\title{
Lipopolysaccharide recognition by human surfactant protein D
}

\author{
William Neale ${ }^{1}$, Jens Madsen ${ }^{2}$, Howard Clark ${ }^{2}$, Annette Shrive ${ }^{1}$, Trevor Greenhough $^{1}$ \\ ${ }^{1}$ School of Life Sciences, Keele University, Newcastle-under-Lyme, ST5 5BG, United Kingdom, \\ ${ }^{2} E G A$ Institute for Women's Health, University College London, WC1E 6HX, United Kingdom
}

w.a.neale@keele.ac.uk

Surfactant Protein D (SP-D) is a member of the collectin family of proteins and acts as part of the innate immune system, the first line of defence, in the lung. The collectins recognise and bind specific structural features conserved amongst pathogens, particularly bacterial and fungal cell surface lipopolysaccharides (LPS) and viral glycans. SP-D can immobilise and form aggregates of pathogens that are more recognisable to neutrophils, along with triggering the rest of the immune system. High-resolution ligand-bound crystal structures of a biologically and therapeutically active recombinant homotrimeric fragment of native human SP-D (rfhSP-D) complexed with simple disaccharides have shown ligand binding to take place through the coordination of a $\mathrm{Ca}^{2+}$ ion and binding site residues to a mannose-type O3' and O4' pair of hydroxyls of the bound sugar [1].

Further work has taken place with fragments isolated from the lipopolysaccharide (LPS) of Haemophilus influenzae Eagan and Salmonella enterica Minnesota R5 through mild acid hydrolysis to cleave the bulky, hydrophobic lipid A [2,3]. These showed preferential binding by rfhSP-D of the LPS inner core through coordination of the calcium to the Hep O6' and O7' sidechain hydroxyls. These ligand-bound structures also highlight that hSP-D has the flexibility and versatility to recognise alternative LPS epitopes when the preferred core heptose is unavailable. In one subunit (subunit A) of the Salmonella LPS bound structure, the proximity of a crystal contact prevents the preferred inner core binding mode seen in the other chains, resulting in binding through the terminal glucose.

Crystals were also frozen at varying time-stages of the ligand soak, and analysis of these structures provides insight into the changes in the interaction between the protein and ligand over time as binding with the LPS progresses. The definition of the bound ligand in the electron density is seen to increases as the soaking time progresses. Comparison of the crystal structures over time also shows that in chain B the non-bound terminal glucose of the oligosaccharide, initially aligned planar with Pro319, rotates by $90^{\circ}$ around the GlcHepII glycosidic bond as binding progresses. There are also structural changes as depletion of the tertiary calcium site progresses over the time course of the ligand soak.

[1] Shrive AK, Tharia HA, Strong P, Kishore U, Burns I, Rizkallah PJ, et al. (2003) 'High-resolution Structural Insights into Ligand binding and Immune Cell Recognition by Human Lung Surfactant Protein D'. J Mol Biol. 331(2):509-23

[2] Littlejohn JR, da Silva RF, Neale WA, Smallcombe CC, Clark HW, Mackay R-MA, et al. (2018) 'Structural definition of hSP-D recognition of Salmonella enterica LPS inner core oligosaccharides reveals alternative binding modes for the same LPS'. PLoS One. 13(6)

[3] Clark, H.W., Mackay, R.M., Deadman, M.E., Hood, D.W., Madsen, J., Moxon, R., Townsend, J.P., Reid, K.B.M., Ahmed, A., Shaw, A.J., Greenhough, T.J., Shrive, A.K. (2016). 'Crystal Structure of a Complex of Surfactant Protein D (SP-D) and Haemophilus influenzae Lipopolysaccharide Reveals Shielding of Core Structures in SP-D-Resistant Strains’. Infection and Immunity, 84 (5), 1585 - 1592.

Keywords: sp-d; lipopolysaccharide; protein; binding 